\title{
INFLUENCE DE L'EXTENSION DU PORT DU HAVRE SUR LES COURANTS DE MAREE
}

\author{
X. LE BARS - Port Autonome du Havre
}

\begin{abstract}
To face the pressure of increasing traffic the Port of Le Havre wishes to extend its tiding docks. The proposed new port layout involves a substantial increase in the plan area of the fidal basins. This will result in larger tidal currents in the port which could adversely affect navigation in the harbour.

A 20 flow model with a uniform grid of $30 \mathrm{~m}$ has been used to investigate the flows in the port both with and without the second entrance. The model demonstrated that the second entrance would not reduce the tidal velocities as hoped but would increase them instead.
\end{abstract}

\section{INTRODUCTION}

Ces cina dernières années, le trafic de conteneurs du Port du Havre a augmenté de près de $50 \%$, atteignant 900000 unités équivalent vingt pieds.

Pour accompagner cette croissance, le Port Autonome du Havre a mis en service en 1990, $1100 \mathrm{~m}$ de nouveaux quais.

En 1993,450 m de quais supplémentaires seront livrés au trafic. Au delà de cette date, les perspectives de développement de l'activité, à l'horizon de l'an 2000. amènent à programmer une extension du port en bassin de marée hors de ses limites actuelles. Ceci devant entrainer des modifications dans le régime des courants de marée, une étude spécifique sur ce point était à mener.

\section{EXPOSE DU PROBLEME}

\subsection{Situtation actuelle}

Développé au fil des siècles depuis 1517, date de sa création par François ler, le Port du Havre peut très schématiquement être divisé en deux parties. 
Une partie de bassins de marée. Celle-ci est en communication directe avec la mer où est établi un régime de marées dont le niveau peut varier entre + $0,30 \mathrm{~m}$ et $+8,30 \mathrm{~m}$ par rapport au zéro des cartes marines locales (CMH). La communication entre les bassins de marées et la mer se fait par l'intermédiaire d'une passe unique de $250 \mathrm{~m}$ de large. Celle-ci commande le régime des courants de marées dans les bassins librement marnant.

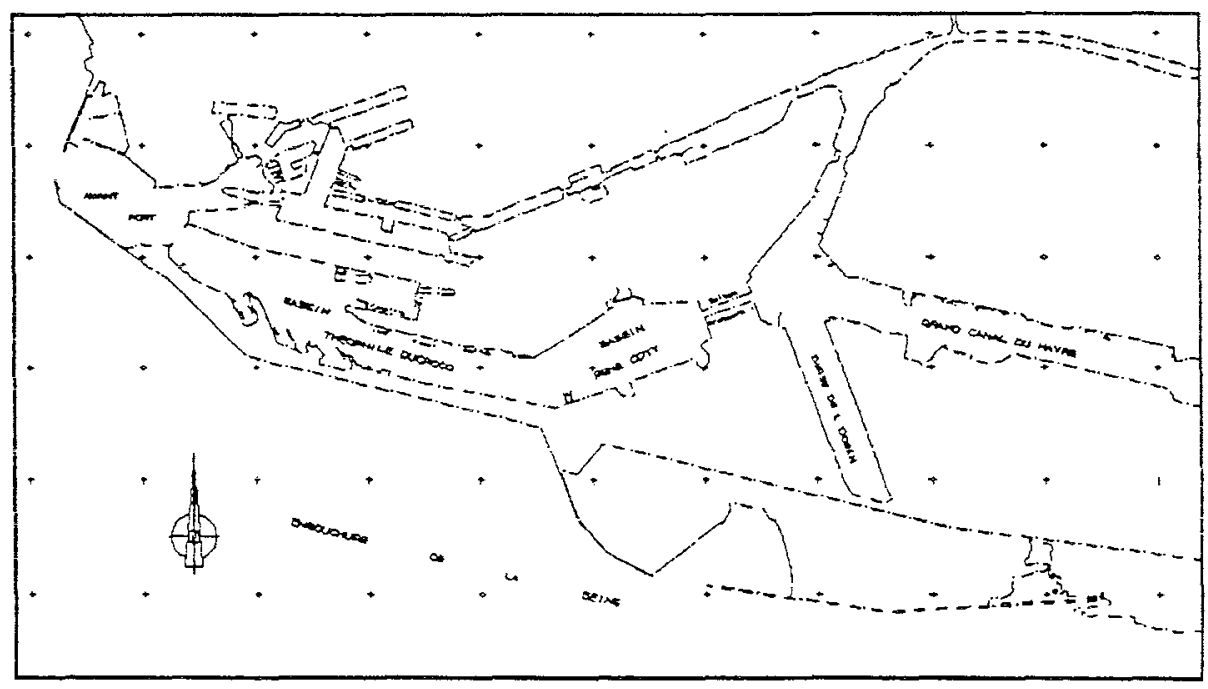

Fig. 1 : Plan d'ensemble

Une partie de bassins à nivequ constant. L'accès à ces bassins depuis les bassins de marée se fait par l'intermédiaire de l'écluse françois jer. Le niveau dans ces bassins est maintenu à une cote d'environ $(+7 \mathrm{CMH})$.

Pour les navires porte-conteneurs modernes, qui représentent des investissements très importants, leurs armements demandent de plus en plus à s'affranchir des contraintes, en particulier de temps, de passage d'une écluse.

Ceci a conduit le Port Autonome du Havre à construire ses nouveaux terminaux à conteneurs en bassin de marée.

En 1990, $1100 \mathrm{~m}$ linéaires de nouveaux quais ont été mis en service. Poursuivant l'accompagnement de la demande, le PAH projette d'équiper de nouveaux sites en bassin de marée. La saturation des sites actuels impose dien rechercher de nouveaux.

\subsection{Nouvelle ouverture du Port}

L'extension du port est prévue pour se développer à moyen terme dans un nouveau bassin, le bassin du Pacifique où dans le courant de lannée 1993 , $450 \mathrm{~m}$ de nouveaux quais seront livrés au trafic. 
Au delà, il est prévu d'étendre le bassin du Pacifique par extension sur l'estuaire de la seine. Le nouveau système de bassins de marée devant entraîner une intensification des courants éventuellement incompatibles avec la sécurité des manoeuvres des navires dans le port, la possibilité d'y remédier par l'aménagement d'une nouvelle entrée sur l'estuaire devrait être étudiée. Cette nouvelle entrée devant conduire à deux accès au port pourrait permettre de ne pas accroître lintensité des courants.

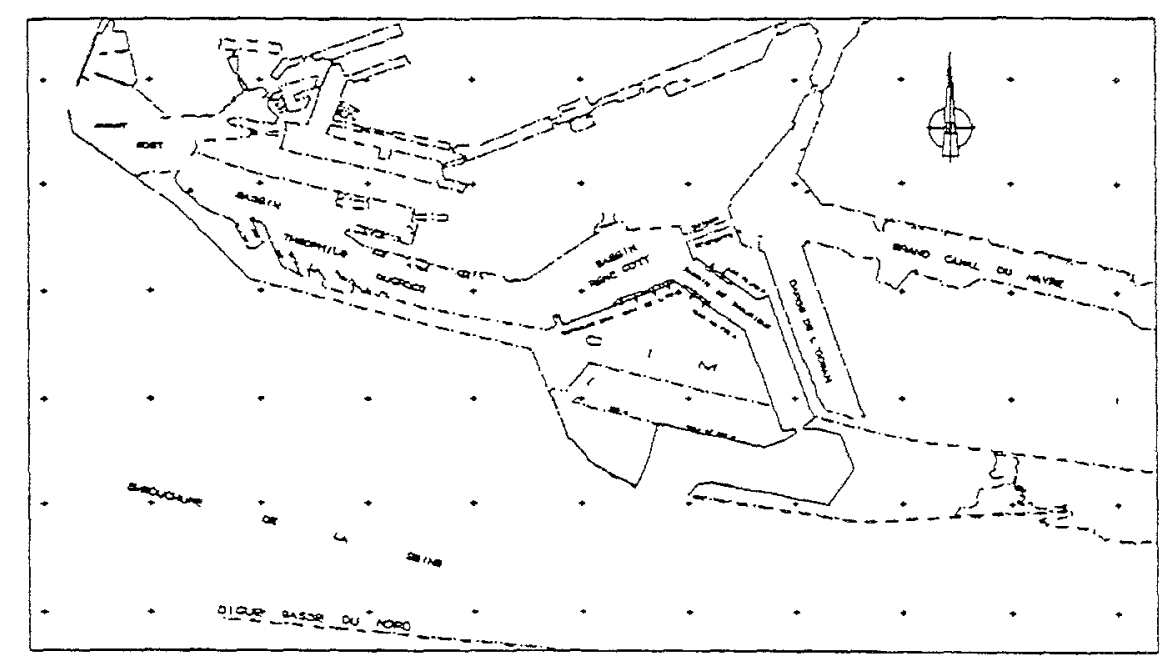

Fig. 2: Extension future du port

\subsection{Courants}

Les courants les plus forts s'établissent actueliement dans la passe d'entrée du port. De quasiment nuls à la basse mer, ils s'élèvent à $0.7 \mathrm{~m} / \mathrm{s}$ environ vers le milieu du fiot pour s'annuler de nouveau à la pleine mer, its reprennent une force de $0.4 \mathrm{~m} / \mathrm{s}$ vers le milieu du jusant. Ceci est observé pour une marée de coefficient 95 . Ils correspondent à des courants longitudinaux. On peut noter également des courants traversiers notamment au droit de l'écluse François ler qui restent compatibles avec la manoeuvre des navires.

\section{LE MODELE MATHEMATIQUEE}

En liaison avec le laboratoire Britannique "Hydraulic Research" de Wallingford un modèle mathématique a été bâti dans le but de simuler les courants.

\subsection{Le modèle}

Le modèle fut bâti sur une grille composée de mailles de $30 \mathrm{~m}$ de côté alignée sur l'écluse François ler. Elle contenait environ 8000 noeuds et permettait de modéliser une décharge de l'écluse si nécessaire. Le nom du modèle est TIDEFLOW-2D. 


\subsection{Les conditions qux limites}

Les concitions cux limites furent appliquées à partir de niveaux de marée mesurés toutes les 15 minutes à chacune des extrémités du modèle. Les évolutions de la marée aux niveaux de l'entrée du port et de la nouvelle entrée sont figurées sur la figure ci-annexée. On y note un décalage d'environ 10 minutes entre les deux courbes.

\subsection{Calage du modèle}

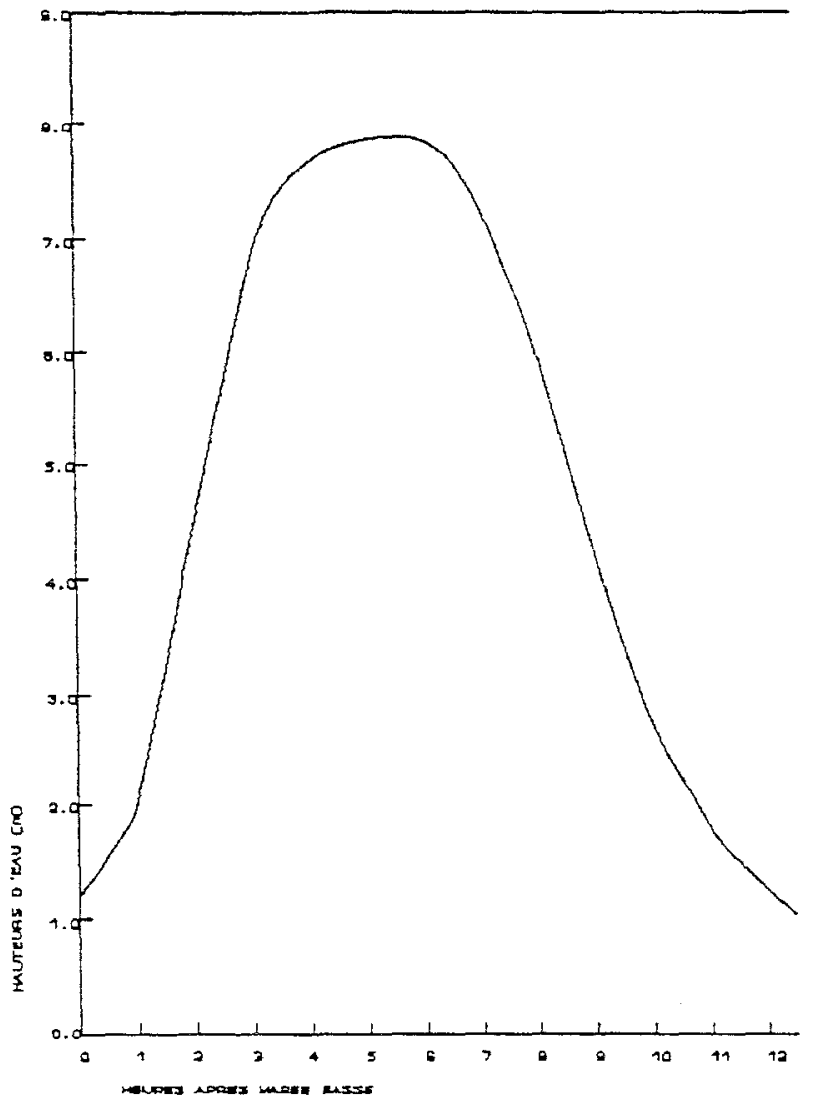

Fig. 3: Courbe de marée

Le modèle a été configuré pour simuler les conditions d'une marée de niveau de coefficient 95 .

Les paramètres d'étalonnage du modèle ont été la rugosité du fond et le coefficient de viscosité des remous. Sur les parois du port une condition de glissement partiel a été utilisée pour simuler la rugosité.

Le modèle a permis de reproduire les caractéristiques essentielles des courants de marée dans le port: sens, phase, amplitude. La simulation a été jugée satisfaisante compte tenu des besoins de l'étude. 


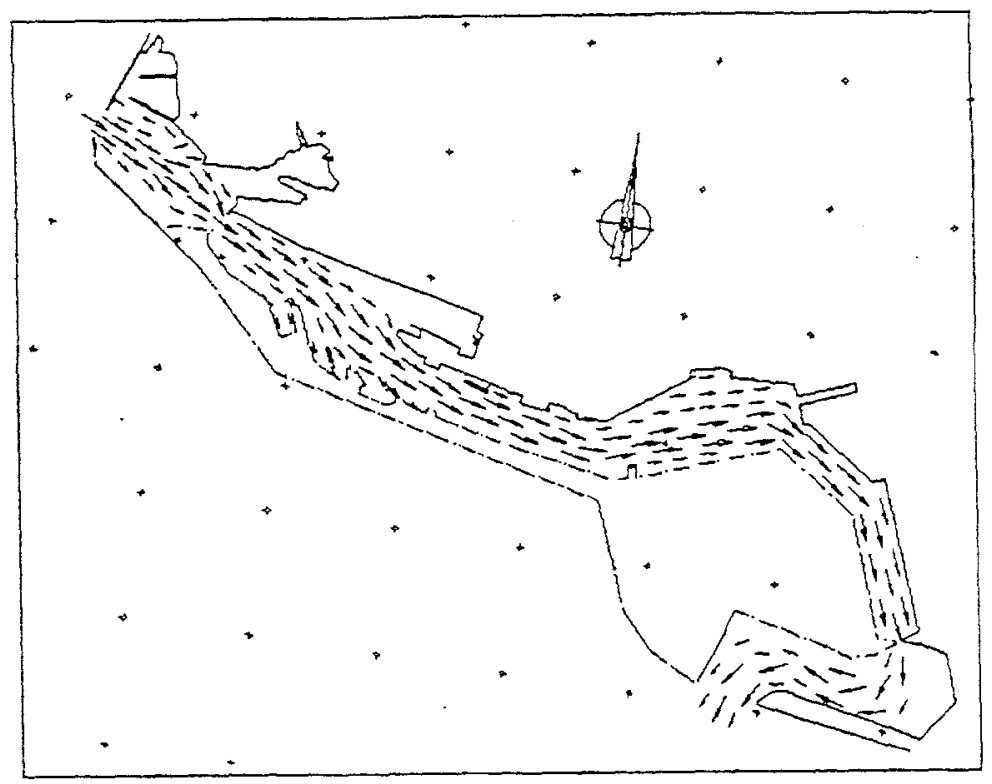

Fig. 4: Courant de flot

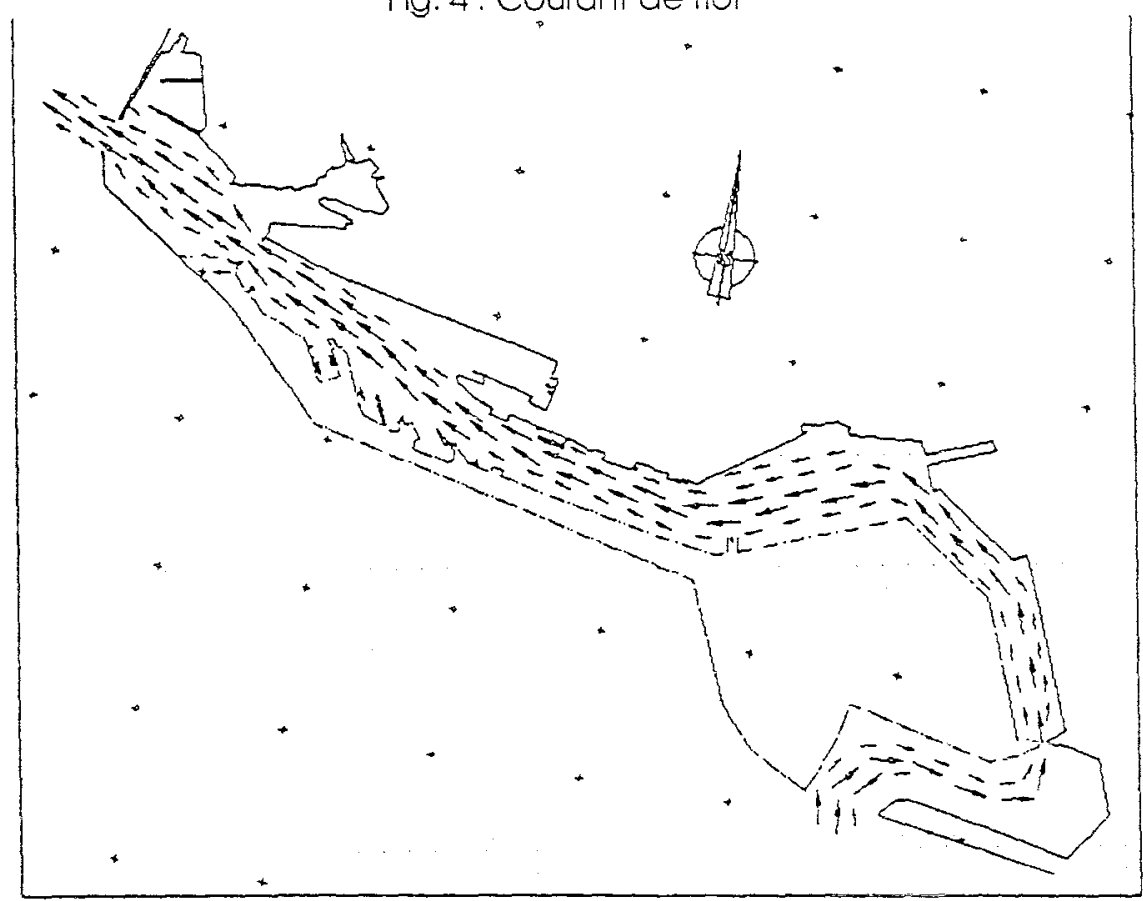

Figure 5: Courant de jusant

\section{LES ESSAIS}

Sur le modèle ainsi calé divers essais ont été menés.

\subsection{Les extensions du port avec deuxième entrée}

Les prévisions du modèle ont montré que les courants de marée croitraient de façon significative comparés à ceux de l'état actuel.

L'augmentation des courants de marée est due au fait que la marée au niveau de la deuxième entrée a environ 10 minutes de retard sur celle de l'entrée du port actuelle. 
Ceci entraine un flux supplémentaire d'eau à travers le port lors de la montée et de la descente de la marée.

\subsection{Extensions du port sans deuxième entrée}

Cet essai a mis en évidence des courants plus faibles que ceux de l'essai précédent.

\subsection{Développement du port par étape}

Un certain nombre dessais supplémentaires ont été développés afin de tester les possibilités de développer le port sans deuxième entrée par phases.

Les résultats obtenus seront d̀ analyser avec les exploitants du port afin de vérifier que les courants obtenus restent compatibles avec la manoeuvre des navires.

\section{CONCLUSION}

Le port Autonome du Havre, pour faire face au développement de son trafic. doit programmer la mise en service à moyen ef long termes de nouveaux quais en bassins de marée. La définition de ces nouveaux bassins pose de délicats problèmes, en particulier de courants.

Le recours à un modèle mathématique hydrodynamique ò deux dimensions a permis de cerner ces problèmes et a apporté de précieux enseignements sur le fonctionnement des configurations envisageables.

Les essais par modèles physiques, quil resteront en tout état de cause nécessaires ultérieurement, seront ainsi allégés et grandement facilités.

\section{REFERENCES BIBLIOGRAPHIQUES}

Port Autonome du Havre

The effect of extending the port on tidal currents

H.R. WALLINGFORD - J. PURIE - DR A.J. COOPER 